\title{
Revisiting an old foe: The face of psychosis in neurosyphilis
}

\author{
Y Moolla, MB ChB, FCP (SA), MMed, Dip HIV Man (SA); J Abdul, MB ChB \\ Department of Internal Medicine, Addington Hospital, Durban, South Africa
}

Corresponding author: Y Moolla (moollayusuf@hotmail.com)

\begin{abstract}
A delusional, agitated middle-aged man presented to hospital with a tenacious psychotic episode. Upon appropriate therapy for neurosyphilis, dramatic resolution of this brief episode ensued, prompting a literature review of psychosis associated with neurosyphilis.
\end{abstract}

S Afr Med J 2016;106(12):1186-1187. DOI:10.7196/SAMJ.2016.v106i12.11446

Syphilis, a sexually transmitted disease caused by Treponema pallidum, continues to be a considerable health burden worldwide. It affects over 30 million people, the majority of whom are from Africa and South-East Asia. ${ }^{[1]}$

This disease, which is also known as the 'great imitator', has three stages. Primary syphilis occurring at the site of inoculation may present as a painless macule which often later ulcerates. The secondary stage usually occurs 4-8 weeks later and may manifest with a generalised rash involving the palms and soles of the feet. Accompanying systemic vasculitis can cause a wide variety of syndromes, which include hepatitis, iritis, nephritis and neurological abnormalities. Left untreated, approximately $30 \%$ of affected individuals will go on to develop tertiary syphilis. The cardinal manifestations of this late form of syphilis are cardiovascular, gummatous and neurosyphilis. ${ }^{[2]}$

Neurosyphilis has a wide array of clinical manifestations; the different pathological subtypes are shown in Table 1. Classic presentations of neurosyphilis are now becoming less common, as recent literature describes cases where psychiatric or neurocognitive symptoms are the solitary signs of the disease. ${ }^{[3]}$ Except in the context of HIV infection, the diagnosis of neurosyphilis is generally considered unusual. Neurosyphilis may develop at any time in the course of T. pallidum infection, and while classic late syphilis manifested 5 - 25 years later in the pre-penicillin era, early meningovascular syphilis may develop within months to years following infection. ${ }^{[4]} \mathrm{A}$ paucity of available data remains regarding the prevalence of neurosyphilis among psychiatric

\section{Table 1. Stages of neurosyphilis}

\begin{tabular}{ll}
\hline Stage & Symptoms \\
\hline $\begin{array}{l}\text { Symptomatic } \\
\text { Early }\end{array}$ & None \\
& Meningeal \\
Late & Meningovascular \\
& Gummatous \\
& Cerebral \\
& Spinal compression \\
& Parenchymatous \\
& General paresis \\
& Tabes dorsalis \\
& Optic atrophy
\end{tabular}

patients in South Africa (SA). A recent study demonstrated that the prevalence of syphilis was $11.7 \%$ among psychiatric patients at a state psychiatric hospital. ${ }^{[5]}$

\section{Case report}

A 52-year-old man was escorted to our regional hospital by local police. The history provided during this involuntary consultation was that of aggression towards his family. The patient's family had noticed a change in his personality, together with blunted emotional responses over the past few weeks. No history of chronic medical disease or of substance abuse was found. Our patient reported feeling well and repeatedly said that he could communicate with God.

On clinical examination, there were no signs of trauma or alcoholism. He was not wasted, and signs of nutritional deficiency were absent. Stigmata of endocrine diseases were absent and normal systemic examination findings were evident. The central nervous system examination was normal; in particular no signs of meningitis or cranial nerve neuropathies were present, pupil reflexes were normal and he had a normal gait. At first consultation we were unable to perform a mini-mental test and despite the use of typical antipsychotic therapy, the delusional state together with mild aggression had persisted.

Biochemical and haematological markers were normal; in addition the C-reactive protein was low, thyroid function test normal and urine drug screen clear. Further investigations revealed a normal brain computed tomography (CT) scan, and a non-reactive HIV test. A lumbar puncture was then performed which prompted the performance of a rapid plasmin reagent (RPR).The results are listed in Table 2. Our patient was diagnosed with neurosyphilis and was started on penicillin G 24 million units per day intravenously, and he continued to receive a high dose of haloperidol. A repeat lumbar puncture performed on day 14 demonstrated a greater than fourfold decrease in cerebrospinal (CSF) Venereal Disease Research Laboratory (VDRL) titre, normalisation of lymphocyte count and CSF protein. Complete resolution of psychosis had occurred, and our patient was discharged.

\section{Discussion}

This case highlights how syphilis lives up to its name the great imitator' and, as illustrated, psychosis may be the sole sign of the disease. Psychosis is often associated with syphilis. Danielsen et al ${ }^{[6]}$ analysed 92 patients with neurosyphilis and $17 \%$ had presented with 
Table 2. Results of investigations

\begin{tabular}{ll}
\hline Syphilis screen & RPR + (titre $>32)$ \\
Lumbar puncture & Polymorphs: 0; lymphocytes: 56; red blood cells: 0 \\
& Protein 0.92 g/L; glucose 3.3 mmol/L (serum 5.1) \\
& VDRL + (titre 128); TPHA + herpes simplex virus; PCR: negative (CSF) \\
\end{tabular}

TPHA $=$ Treponema pallidum haemagglutination; $\mathrm{PCR}=$ polymerase chain reaction.

psychiatric symptoms, while Timmermans and Carr $^{[7]}$ described $51 \%$ of 161 patients having had neuropsychiatric manifestations. It has been cited that the most common presenting feature was personality change; however, memory impairment, hostility, confusion, hallucination and delusions may also occur in neurosyphilis. ${ }^{[8]}$ These presenting signs should prompt a screening RPR. Furthermore, a study by Lin $e t$ al ${ }^{[3]}$ found that psychiatric manifestations were the primary symptoms of neurosyphilis in 52\% of 169 patients.

Numerous case reports further add to the minefield of neuropsychiatric manifestations associated with this disease, psychotic symptoms with hypomania, depression with psychotic features and somatic preoccupation, dementia with psychotic features, and treatment-resistant psychosis, to name a few. ${ }^{[9-12]}$ Our case adds to these and parallels this change of face of modern neurosyphilis, but more importantly demonstrates that rapid clinical improvement and reversibility of the neuropsychiatric burden following antibiotic therapy is attainable. This phenomenon has been previously documented in patients with comparable presentations and further highlights the importance of a prompt diagnosis together with appropriate timely therapy. ${ }^{[13,14]}$ While neither guideline nor consensus exists on the neurotropic agents of choice, Sanchez and Zisselman ${ }^{[15]}$ in a case series support the utilisation of psychotropic medication in combination with antibiotic therapy. Haloperidol, atypical agent risperidone and quetiapine appeared to have provided clinical benefit. Anti-epileptic therapy also appeared to assist in mood stabilisation. Using the lowest effective dose is supported, together with periodic attempts to reduce or withdraw therapy. ${ }^{[15]}$

The atypical forms of neurosyphilis now seen may be attributed to the widespread use of beta-lactamase antibiotics and HIV co-infection. ${ }^{[16]}$ Being in the midst of the HIV epidemic, clinicians in Africa should take heed of the re-emergence of this disease and have a heightened clinical suspicion. The prevalence of neurosyphilis among patients with HIV infection may be as high as $23.5 \%$. HIV co-infection with syphilis is a symbiotic one, as syphilis infection is able to facilitate HIV transmission, while HIV allows for unusual and aggressive manifestations, which includes early neurological involvement. ${ }^{[17]}$ Diagnosis may prove to be challenging, with higher rates of serological negative tests and increased false-negative nontreponemal tests secondary to the prozone effect while syphilis persistence following therapy is an added concern. ${ }^{[18]}$

\section{Conclusion}

While HIV has altered both the prevalence and presentation of neurosyphilis, the importance of routine non-treponemal testing as a screening tool for syphilis is vital in a psychotic patient regardless of HIV status. We emphasised its use together with further invasive investigations such as a lumbar puncture with CSF-VDRL testing in cases where the suspicion of neurosyphilis is heightened. Direct admission to a psychiatric unit should be avoided, as this may cause undue delay in the diagnosis and result in unnecessary investigations together with misuse of resources. Unlike the leopard phenotype which ceases to change, this chameleon of a disease continues to pose a challenge to both physician and psychiatrist.

Wridteath Infections - 2008-2012. http://www.who.int/reproductivehealth/publications/rtis/stisestimates/en/

2. French P. Syphilis. BMJ 2007;334:143-137. http://dx.doi.org/10.1136/bmj.39085.518148.BE

3. Lin L, Zhang H, Huang S, et al. Psychiatric manifestations as primary symptom of neurosyphilis among HIV-negative patients. J Neuropsychiatry Clin Neurosci 2014;26(3):233-240. http://dx.doi. org/10.1176/appineuropsych.13030064

4. Marra CM. Neurosyphilis. Continuum (Minneap Minn) 2015;21(6):1714-1728. http://dx.doi.org/10.1212/ con. 0000000000000250

5. Henning MP, Krüger C, Fletcher L. Syphilis sero-positivity in recently admitted and long-term psychiatric inpatients: Screening, prevalence and diagnostic profile. S Afr J Psychiatr 2012;18(4):171-175. psychiatric inpatients. Screening,

6. Danielsen AG, Weismann K, Jorgensen BB, Heidenheim M, Fugleholm AM. Incidence, clinical 6. Danielsen AG, Weismann K, Jorgensen BB, Heidenheim M, Fugleholm AM. Incidence, clinical
presentation, and treatment of neurosyphilis in Denmark, 1980-1997. Acta Derm Venereol presentation, and treatment of neurosyphilis in Denmark,

7. Timmermans M, Carr J. Neurosyphilis in the modern era. J Neurol Neurosurg Psychiatry 2004;75(12):1727-1730. http://dx.doi.org/10.1136/jnnp.2004.03192

8. Roberts MC, Emsley RA. Psychiatric manifestations of neurosyphilis. S Afr Med J 1992;82(5):335-337. Mahendran R. Clozapine in the treatment of hypomania with neurosyphilis. J Clin Psychiatry 2001;62(6):477-478.

10. Mirsal H, Kalyoncu A, Pektas Ö, Beyazyürek M: Neurosyphilis presenting as psychiatric symptoms An unusual case report. Acta Neuropsychiatr 2007;19(4):251-253. http://dx.doi.org/10.1111/j.16015215.2007.00209.x

11. Hutto B. Syphilis in clinical psychiatry. Psychosomatics 2001;42(6):453-460. http://dx.doi.org/10.1176/ appi.psy.42.6.453

12. Van Eijsden P, Veldink JH, Linn FH, Scheltens P, Biessels GJ. Progressive dementia and mesiotemporal atrophy on brain MRI: Neurosyphilis mimicking pre-senile Alzheimer's disease. Eur J Neurol 2008:15:14-e15.

13. Mannekote ST, Singh VK. A case of neurosyphilis presenting with treatment-resistant psychotic symptoms and progressive cognitive dysfunction. German J Psychiatry 2008;11:153-155

symptoms and progressive cognitive dysfunction. German J Psychiatry 2008;11:153-155
14. Crozatti LL, Brito MH, Lopes BNA, Campos FPF. Atypical behavioral and psychiatric symptoms: Neurosyphilis . Crozatti LL, Brito MH, Lopes BNA, Campos FPF. Atypical behavioral and psychiatric symptoms: Neurosyph
should always be considered. Autopsy Case Rep 2015;5(3):43-47. http://dx.doi.org/10.4322/acr.2015.021

should always be considered. Autopsy Case Rep 2015;5(3):43-47. http://dx.doi.org/10.4322/acr.2015.021
15. Sanchez FM, Zisselman MH. Treatment of psychiatric symptoms associated with neurosyphilis. Psychosomatics 2007;48(5):440-445. http://dx.doi.org/10.1176/appi.psy.48.5.440

16. Barry GH, Miriam B. Evolution of the serine $\beta$-lactamases: Past, present and future. Drug Resist Updat 2004; 7(2):111-123. http://dx.doi.org/10.1016/j.drup.2004.02.003

17. Lynn WA, Lightman S. Syphilis and HIV: A dangerous combination. Lancet Infect Dis 2004;4(7):456-466. http://dx.doi.org/10.1016/S1473-3099(04)01061-8

18. Haslett P, Laverty M. The prozone phenomenon in syphilis associated with HIV infection. Arch Intern Med 1994;154(14):1643-1644. http://dx.doi.org/10.1001/archinte.1994.00420140115016

Accepted 30 August 2016. 